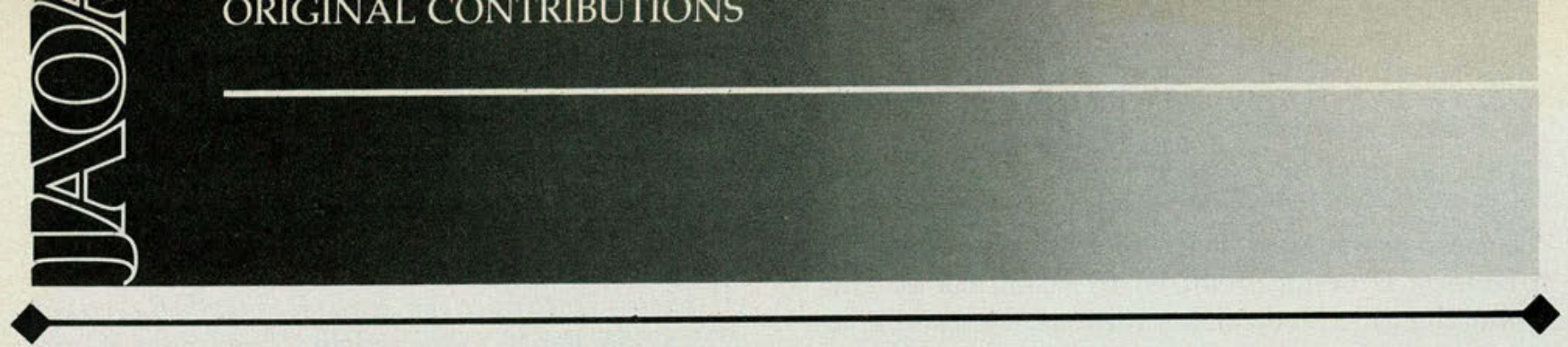

\title{
Evaluation of two manipulative techniques in the treatment of postexercise muscle soreness
}

\author{
D. MOLEA, B.S. \\ B. MURCEK, B.S. \\ C. BLANKEN, B.S \\ R. BURNS, B.S \\ A. CHILA, D.O. \\ J. HOWELL, Ph.D \\ Athens, Ohio
}

To evaluate the effect of manipulative treatment in postexercise muscle soreness (PEMS), soreness was induced in the elbow flexors of 27 healthy, young adults. At 48 hours after the exercise, when the soreness was most severe, subjects were treated with either a myofascial manipulative technique, a muscle energy technique, or a placebo technique. Range of motion and upper arm circumference were measured before exercise, before treatment, immediately aitter treatment, and 48 hours post-treatment. Pain was assessed and recorded by the subjects each morning and evening for 6 days after the exercise. No significant differences appeared among the treatment and control groups, except in the single instance of a transient decrease in arm circumference immediately after treatment with the muscle energy technique. By analyzing present knowledge and theory regarding PEMS, somatic dysfunction, and manipulative techniques, the authors attempt to account for the apparent lack of efficacy of the techniques employed in the study.

Postexercise muscle soreness (PEMS), which also is known as delayed onset muscle soreness, occurs after strenuous and/or infrequent exercise and has been reported as a problem in sports medicine, in occupational and environmental health areas, and in the designing of exercise programs for both rehabilitation and general fitness. ${ }^{1-3}$ PEMS has been characterized by muscular soreness and tenderness that tends to peak 48 hours after exercise and disappear within 5 to 7 days. It also is characterized by weakness, edema, and stiffness (that is, reduced range of motion) in the involved muscles. ${ }^{3-6}$ Static stretching, heat, and mild exercise have been reported to aid in alleviating the symptoms of PEMS. ${ }^{7,8}$ In an earlier study, ${ }^{6}$ we demonstrated that the restriction of motion (stiffness) that occurs in PEMS cannot be accounted for by reflex changes in the proprioceptive control of muscles involved. In this study, we have examined the effects of two manipulative techniques on PEMS and have considered the possible relevance of the results to somatic dysfunction of clinical interest.

\section{Methods}

The subjects included in this study were 27 young adults who were judged to be free of apparent somatic dysfunction after brief medical history taking and structural examination. Exclusion criteria included the following: participation in weight-training programs; history of cardiovascular disease; and traumatic or neurologic disorders involving the neck and upper extremity regions. Childhood fractures of the radius and/or ulna did not exclude potential subjects from participation.

The structural examination consisted of range- 
of-motion (ROM) testing of the neck and upper extremities. Specific measurements included the angles of active and passive elbow extension, the resting angle of the elbow, and the upper arm circumference (UAC). These measurements were taken with the subjects in a standing position. Resting angles were obtained with the entire arm hanging freely. For active extension, subjects were instructed to extend their elbows as far as they could. For passive extension, the examiner extended the elbows to the limit of motion.

ROM measurements are presented in the tables and figures as the supplement to the angle formed by lines joining the lateral epicondyle of the humerus to the acromion of the scapula and to the styloid process of the ulna, with the forearm being maintained in a semiprone position. Thus, the fully extended elbow is indicated by an angle near zero, the supplement of 180 degrees. UAC was measured at three points - the deltoid insertion on the humerus (upper), the midbelly of the biceps (middle), and a point just above the humeral epicondyles (lower).

Pain ratings were obtained with the use of a fourpoint rating scale similar to those used by Abraham $^{1}$ and by DeVries 9 : 0-no pain; 1-pain upon palpation only; 2-pain upon palpation and with movement; and 3 - continuous pain. Pain ratings were obtained at the beginning of the initial examination, which was always performed on a Monday, and for each morning and evening of the following 5 days, that is, through Saturday evening.

PEMS was induced in the elbow flexors of the nondominant arm by means of a regimen of eccentric contractions utilizing loads equal to $80-90$ percent of the maximum isometric force that the subject could exert. Subjects were seated on a specially constructed bench that provided padded support for the arm at 30 degrees from the vertical. Loads were attached via a pulley system to a wristband worn by the subject. The subject was exposed to the load with the elbow near full flexion. He/she then lowered it slowly (over 4-7 seconds) to full elbow extension. The operator then lifted the load and again transferred it to the subject's fully flexed arm. The procedure was repeated until the subject could no longer effectively resist the load and slow its fall; this usually required 12 to 40 repetitions.

The subjects were assigned randomly to treatment groups. The treatment for group $1(n=9)$ was a myofascial technique in which the forearm was flexed as far as the subject could tolerate and pronated by application of a torsional force to the hand and wrist. The arm was then very slowly extended while increasing torque was applied. When the limit of extension was reached, the arm was again flexed at the elbow. It was then hypersupinated by the application of a torsional force to the hand and wrist. The arm was again very slowly extended while increasing torque was applied.

The treatment for group $2(n=10)$ consisted of a muscle energy technique in which the arm was extended to the point of onset of resistance. The subject was then instructed to exert a force against the operator's hand. This isometric force was exerted by the elbow flexors and directed away from the resistance encountered on extension. It lasted 3 to 5 seconds. The treatment was repeated two times. The procedure for group $3(n=8)$ was designed to serve as a placebo and involved holding the supine subject's occiput in the hands of the operator for approximately the same amount of time required for the other techniques ( 1 to $5 \mathrm{~min}$ utes).

The techniques as well as the examinations just described were performed by the three participating student physicians (D.M., B.M., and C.B.). The procedures corresponded to manipulative techniques employed by osteopathic physicians. However, their use was not based on complete structural diagnoses of the subjects, nor were they carried out to a particular, desired therapeutic endpoint. Thus, they are actually procedures or techniques, not treatments.

The study was designed as a single-blind study in which the examinations and treatments of subjects were carried out by different persons. The examiner was unaware of which treatment group the subjects were in, and the operators who performed the treatments were kept unaware of the results of preceding examinations. For consistency, all of the examinations of a given subject were carried out by the same operator. The overall temporal design of the experiment can be seen in Figure 1.

The data were tested for homogeneity of variance to determine if parametric procedures were appropriate. ${ }^{10,11}$ The pain rating and ROM data satisfied this condition ( $p=.01$ ) and were subsequently analyzed with a split-plot factorial design. Analyses of the UAC data were performed with the use of the nonparametric sign test, ${ }^{12}$ because homogeneity of variance did not hold.

\section{Results}

The pain data were handled in two steps. First, the pretreatment pain ratings were compared to establish that a change had occurred as a result of the exercise regimen and that the treatment group showed no differences prior to treatment. There was a significant increase $(p=.05)$ in the pain ratings over 48 hours following exercise, but, as expected, there were no differences between the 
treatment groups (because no treatment had yet been applied). Second, analysis of the post-treatment data showed a significant decrease $(p=.05)$ in the pain ratings as a function of time after treatment. However, again, no differences between the treatment groups were observed (Fig. 2).

Analysis of the ROM data focused on alterations in passive extension, active extension, and resting angles. The pretreatment data were analyzed first to confirm that changes had occurred due to exercise and that no differences between the groups appeared prior to treatment. Significant changes had occurred in all three angles $(p=.05)$ as seen in Table 1: no differences were noted between groups prior to treatment. The post-treatment data revealed significant decreases in all three angles as a function of time, but, again, there were no differences between the treatment groups (Table 2, Fig. 3).

The pretreatment UAC data revealed significant increases in UAC as a result of exercise for all three positions on the arm, with the greatest changes occurring in the middle $(1.36 \mathrm{~cm}$.) and lower $(1.26$ $\mathrm{cm}$.) measurement sites. The post-treatment data were analyzed for both immediate (that is, before treatment minus after treatment) and 48-hour effects of individual treatments. The only significant change observed ( $p=.05)$, according to the nonparametric sign test, was an immediate decrease in the middle position UAC at the midbelly of the biceps brachii muscle associated with the muscle energy technique (Table 3 ). No other changes occurred with respect to treatment or position. Comparison of before-exercise values with final values indicated that UAC did not return completely to control values during the 96 hours of measurement postexercise $(p=.05)$.

\section{Discussion}

In this study we have used the phenomenon of PEMS as a model for somatic dysfunction. According to the "Glossary of osteopathic terminology,"13 somatic dysfunction is defined as follows: "Impaired or altered function of related components of the somatic (body framework) system: skeletal, arthrodial, and myofascial structures, and related vascular, lymphatic, and neural elements." PEMS seems to fit this definition. The condition involves

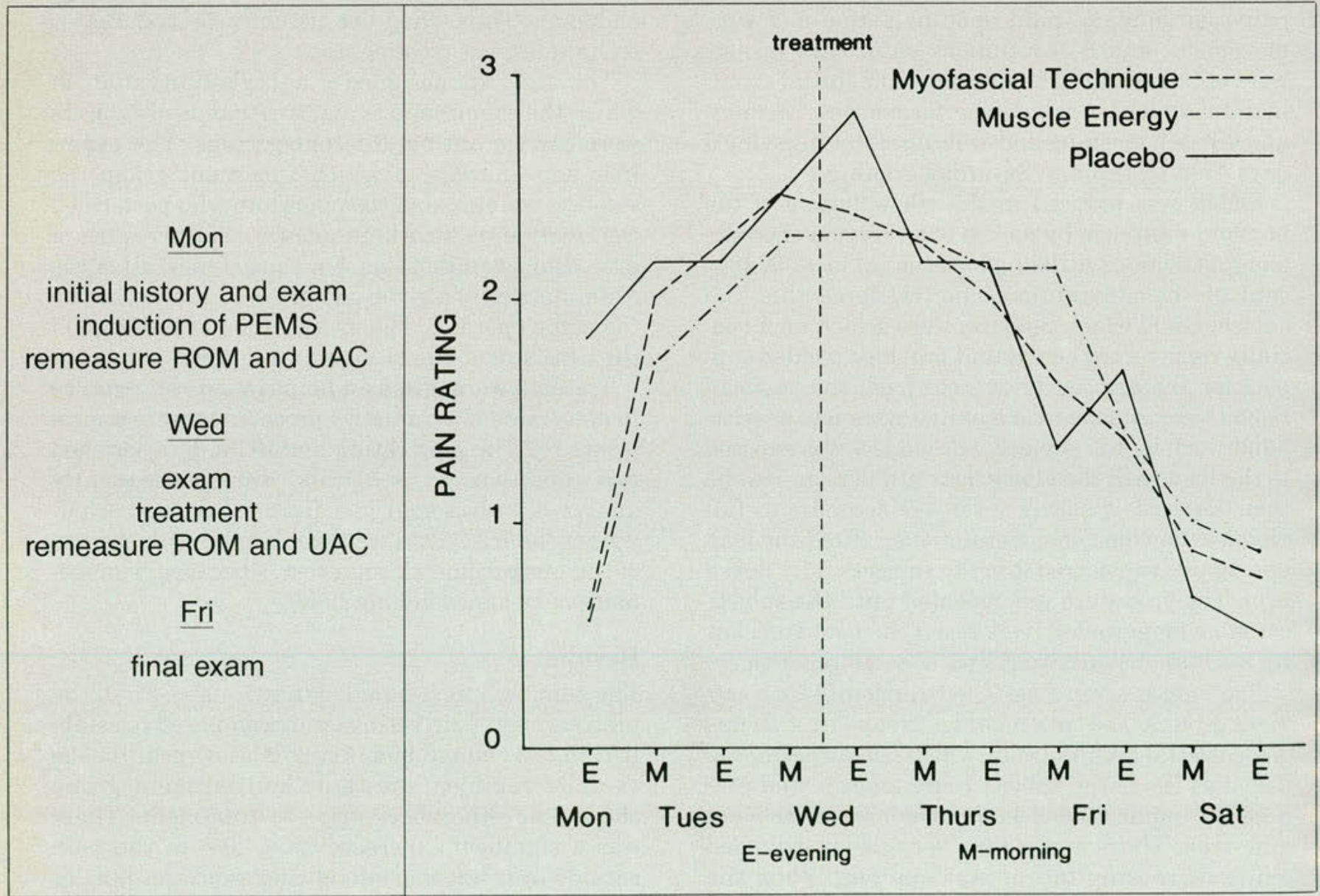

Fig. 1. Temporal aspects of experimental design. Fig. 2. Course of pain over time for all three treatments ( $E$ - evening; $M-m o r n i n g)$. 
demonstrable alterations in joint and muscle function, and the swelling that occurs indicates changes in vascular and/or lymphatic elements. It is not clear that any change in bone function per se occurs, and the extent of neural changes may be limited to the sensation of pain.

With the idea that PEM might serve as a model for somatic dysfunction, we compared two manipulative techniques with a placebo in the treatment of PEMS. With respect to pain, restriction of motion, or edema (increased UAC being indicative of edema), neither technique produced a significant effect on the condition, except in the single instance of reduced UAC in the midbelly of the biceps brachii.

The apparent lack of efficacy of the treatments may to some extent be attributed to difficulties in experimental design. One problem is the use of student physicians in administering the treatments. Questions can be raised about how the technical skill of the operators affects the efficacy of treatment. However, in this study we have defined in precise mechanical terms the procedures used for administering the treatments. As a result, conclusions drawn from the data apply only to the techniques described here.

A second difficulty relates to the problem of how the method of measurement may actually alter what is being measured. This has particular significance for the use of repeated measurements as employed in this study. Measurements were taken for active extension, passive extension, and resting angles at the elbow. Measurement of resting angle probably does not affect ROM, because it is made with the arm completely relaxed. On the other hand, measurements of active or passive extension result in the stretching of the flexors by either the operator (passive) or the subject (active). This stretching can, in effect, be a treatment. As mentioned in the introduction, static stretching has been reported to alleviate the symptoms of PEMS. This problem clearly did not prevent us from seeing the restriction caused by the exercise, but it may have limited our ability to detect modest differences induced by the treatments. Even so, it is unlikely that we would have missed major treatment effects.

In the study of any therapeutic regimen, the basic question that must be asked is whether the regimen is appropriate for the problem it is designed to treat. To appreciate how the treatment and the problem are related, one must look at the theoretic basis of the treatment and compare it with the pathogenic mechanisms underlying the problem.

Explanations of both the muscle energy and myofascial techniques have been offered on the theoretic basis provided by Korr. ${ }^{14} \mathrm{He}$ proposed that the clinical entity of somatic dysfunction can be explained by inappropriately high settings of muscle spindle sensitivity via an increased output of the gamma motor neurons regulating those spin-

\begin{tabular}{|c|c|c|c|}
\hline $\begin{array}{l}\text { Type of } \\
\text { Value* }^{*}\end{array}$ & $\begin{array}{c}\text { Active } \\
\text { extension angle } \\
\text { (degrees) }\end{array}$ & $\begin{array}{c}\text { Passive } \\
\text { extension angle } \\
\text { (degrees) }\end{array}$ & $\begin{array}{c}\text { Resting } \\
\text { angle } \\
\text { (degrees) }\end{array}$ \\
\hline$\overline{\mathrm{BE}}$ & 2.99 & 2.51 & 18.02 \\
\hline$\overline{\mathrm{BT}}$ & 19.99 & 13.76 & 42.69 \\
\hline$\overline{\mathrm{BT}}-\overline{\mathrm{BE}}$ & 17.00 & 11.25 & 24.67 \\
\hline
\end{tabular}

\begin{tabular}{|c|c|c|c|}
\hline $\begin{array}{l}\text { Type of } \\
\text { Value* }\end{array}$ & $\begin{array}{c}\text { Active } \\
\text { extension angle } \\
\text { (degrees) }\end{array}$ & $\begin{array}{c}\text { Passive } \\
\text { extension angle } \\
\text { (degrees) }\end{array}$ & $\begin{array}{c}\text { Resting } \\
\text { angle } \\
\text { (degrees) }\end{array}$ \\
\hline$\overline{\mathrm{BT}}$ & 19.99 & 13.76 & 42.69 \\
\hline$\overline{\mathrm{AT}}$ & 17.59 & 12.40 & 37.27 \\
\hline$\overline{\mathrm{F}}$ & 9.90 & 6.52 & 29.32 \\
\hline$\overline{\mathrm{BT}}-\overline{\mathrm{AT}}$ & 2.40 & 1.36 & 5.42 \\
\hline$\overline{\mathrm{BT}}-\overline{\mathrm{F}}$ & 10.09 & 7.24 & 13.37 \\
\hline
\end{tabular}


dles. Spindle sensitivity is normally regulated by the central nervous system (CNS) to maintain a continuous flow of useful information from the spindles during muscle shortening. ${ }^{15}$
Korr ${ }^{14}$ suggested two situations in which inappropriately high gain might be induced: "(1) strong centrally ordered contraction during a moment (2) when the muscular attachments...have been

\begin{tabular}{|c|c|c|c|c|c|c|}
\hline \multirow[b]{2}{*}{ Subject } & \multirow[b]{2}{*}{ BT } & \multicolumn{2}{|c|}{ Circumference $(\mathrm{cm})}$. & \multicolumn{3}{|c|}{ Circumference $(\mathrm{cm})}$. \\
\hline & & AT & $\mathrm{BT}-\mathrm{AT}$ & BT & F & $B F-F$ \\
\hline 1 & 35.4 & 35.0 & 0.4 & 35.4 & 34.0 & 1.4 \\
\hline 2 & 39.5 & 39.3 & 0.2 & 39.5 & 40.0 & -0.5 \\
\hline 3 & 33.5 & 33.0 & 0.5 & 33.5 & 32.0 & 1.5 \\
\hline 4 & 23.5 & 23.2 & 0.3 & 22.2 & 23.4 & -1.2 \\
\hline 5 & 28.9 & 28.7 & 0.2 & 27.4 & 27.5 & -0.1 \\
\hline 6 & 31.0 & 30.2 & 0.8 & 34.9 & 34.6 & 0.3 \\
\hline 7 & 26.5 & 26.0 & 0.5 & 31.0 & 28.8 & 2.2 \\
\hline 8 & 22.2 & 22.2 & 0.0 & 28.9 & 28.8 & 0.1 \\
\hline 9 & 27.4 & 27.4 & 0.0 & 23.5 & 24.5 & -1.0 \\
\hline 10 & 34.9 & 34.9 & 0.0 & 26.5 & 26.5 & 0.0 \\
\hline
\end{tabular}

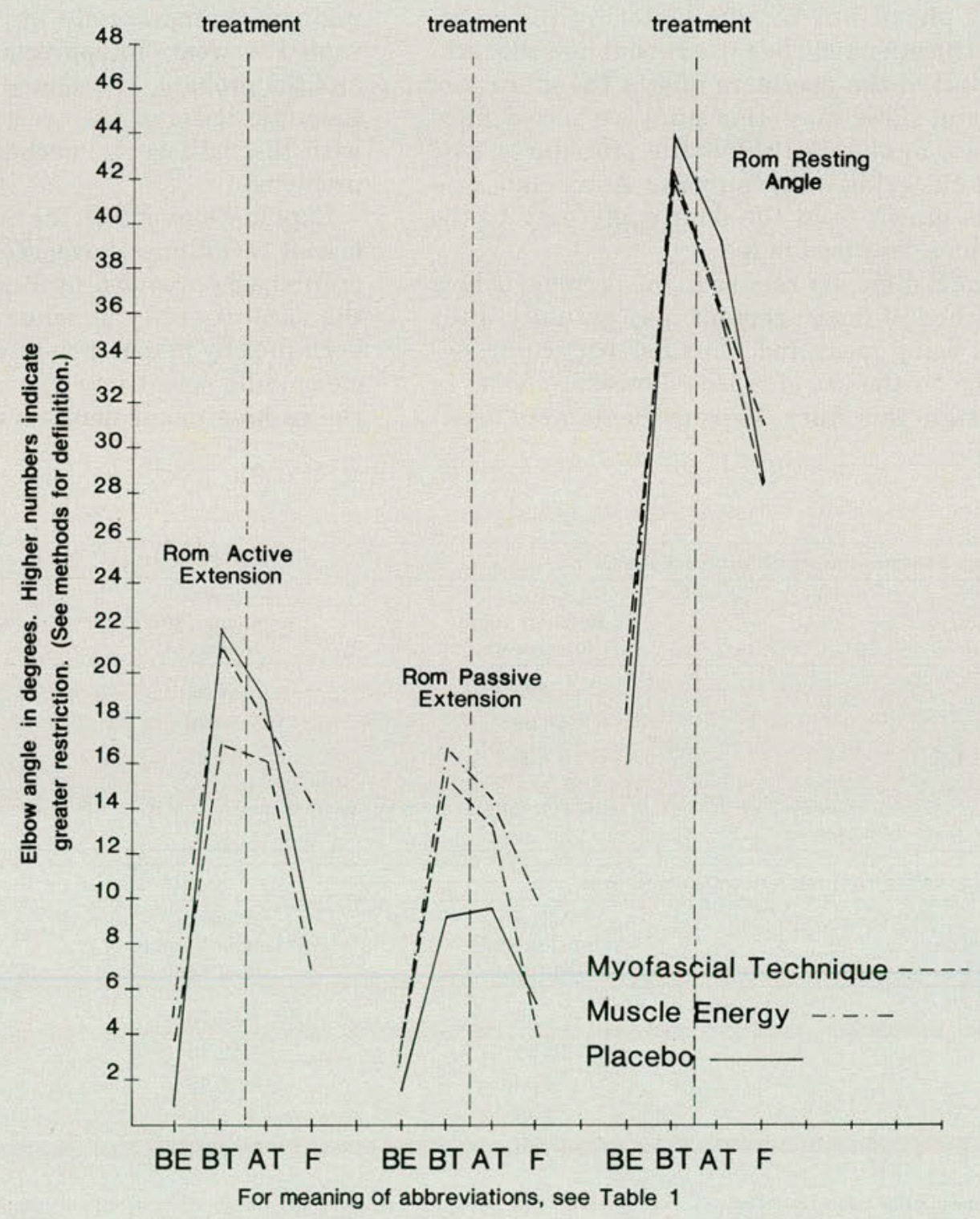

Fig. 3. Active extension, passive extension, and resting angles over time. 
closely and abruptly approximated by forces or factors that have not been centrally ordered." The mechanism by which strong contraction stimulates the CNS to adjust spindle sensitivity is not discussed. On the other hand, it is suggested that the sudden loss of information entering the CNS as a result of the sudden shortening of the muscle stimulates it to increase the spindle sensitivity to regain that informative input. Thus, a key element in producing a change in sensitivity seems to be a sudden and unexpected event to which the CNS cannot respond appropriately. If the CNS already knows what is going to happen and that is what, in fact, does happen, the CNS does not have to respond to a sudden loss of information, because it received no surprises. Therefore, no inappropriate alterations in spindle sensitivity would be expected.

Korr ${ }^{14}$ further proposed that the ability of manipulative therapy to alleviate symptoms of somatic dysfunction, in particular restriction of motion, may result from a resetting of the muscle spindle sensitivity to a more appropriate level. He suggested that three typical manipulative procedures might cause this resetting. They are: (1) gentle shortening of the affected muscles (movement in the direction of ease), which may operate to bring the length of the extrafusal fibers closer to that of the hyperactive intrafusal fibers; (2) stretching of the affected muscles, which may cause resetting via activation of the Golgi tendon organs or by intense activation of the already hyperactive muscle spindle stretch receptors; and (3) force development through isometric contractions at a variety of muscle lengths, which may, like the second kind of manipulative procedure, act either through the Golgi tendon organs or the muscle spindles or both. The myofascial technique employed in this study clearly contained elements of the first and second procedure described, whereas the muscle energy technique corresponds closely to the third kind of procedure.

Both of these techniques may produce local effects in addition to these CNS-mediated reflex changes. For instance, it has been suggested that the myofascial techniques may also produce some effect on fluid distribution, particularly in connective tissue. This may result from the stretching of connective tissue, ${ }^{16}$ which may provide a "milking action" within the connective tissue that forces fluid out of the tissues. Our observation of a decrease in UAC following application of the muscle energy technique suggests that gentle isometric contractions may contribute to the movement and resorption of fluid causing the edema.

An understanding of whether either of these treatments should be helpful in PEMS can be gained by looking at how PEMS is produced-in this study, by strong, eccentric contractions. The contractions were performed in such a way that the subject received no surprises while performing the exercise. $\mathrm{He} / \mathrm{she}$ was told in advance what was going to happen, and that is exactly what did occur. Therefore, there is no reason to believe that the CNS received any surprise to which it had to adjust, and, in accordance with the preceding discussion, no alteration in spindle sensitivity would be expected. Consequently, inappropriate setting of spindle sensitivity would not be expected to contribute to PEMS. Experimental investigations into the possibility that elevated neuromuscular activity occurs in PEMS have not resulted in strong evidence in favor of this idea. DeVries ${ }^{17}$ reported increases in EMG activity during PEMS, but subsequent studies ${ }^{1,18,19}$ have not supported that conclusion. Our earlier work ${ }^{6}$ demonstrated specifically that the restriction of motion of PEMS could not be accounted for by neurally mediated activity in the affected muscles.

In the light of these considerations, neither of the techniques used in this study would be expected to produce any neurally mediated effects on PEMS, because no abnormal alterations in spindle sensitivity are present to be reset; study results bore this out. The transient reduction in UAC in the belly of the biceps muscle immediately after the application of a muscle energy technique is probably the result of local effects influencing fluid distribution.

\section{Conclusions}

The present study confirms the PEMS-associated restriction of motion described in our 1985 work $^{6}$ and illustrates the concept that there may be multiple pathophysiologic mechanisms underlying the clinical conditions that can be referred to as somatic dysfunction. We suggest that an understanding of these pathophysiologic processes may, in the future, help to define various types of somatic dysfunction and may be of value in the choice of appropriate treatment procedures. However, present knowledge is very limited and must be developed further to become useful in the clinical setting.

1. Abraham, W.M.: Factors in delayed muscle soreness. Med Sci Sports 9:11-20, Spr 77

2. Fridén, J.: Muscle soreness after exercise. Implications of morphological changes. Int J Sports Med 5:57-66, Apr 84

3. Talag, T.: Residual muscular soreness as influenced by concentric, eccentric, and static contractions. Res Quart 44:458-69, 1973

4. Asmussen, E.: Observations on experimental muscular soreness. Acta Rheum Scand 2:109-16, 1956

5. Hough, T: Ergographic studies in muscular soreness. Am J Physiol 7:76-92, 1 Apr 02 
6. Howell, J.N., et al.: An electromyographic study of elbow motion during postexercise muscle soreness. J Appl Physiol 58:1713-18, May 85

7. DeVries, H.A.: Prevention of muscular distress after exercise. Res Quart 32:177-85, 1961

8. Armstrong, R.B.: Mechanisms of exercise-induced delayed onset muscular soreness. A brief review. Med Sci Sports Exerc 16:529-38, Dec 84 9. DeVries, H.A.: Quantitative electromyographic investigation of the spasm theory of muscle pain. Am J Phys Med 45:119-34, Jun 66

10. Steinmetz, J.E., Romano, A.G., and Patterson, M.M.: Statistical programs for the Apple II microcomputer. Behav Res Meth Instrument 13:702, Oct 81

11. Winer, B.J.: Statistical principles in experimental design. Ed. 2. McGraw-Hill Book Co., New York, 1971

12. Minium, E.W., and Clarke, R.B.: Elements of statistical reasoning. John Wiley \& Sons, Inc., New York, 1982

13. Glossary of osteopathic terminology. JAOA 80:552-67, Apr 81

14. Korr, I.M.: Proprioceptors and somatic dysfunction. JAOA 74:638-50, Mar 75

15. Loeb, G.E., Hoffer, J.A., and Pratt, C.A.: Activity of spindle afferents from cat anterior thigh muscles. I. Identification and patterns during normal locomotion. J Neurophysiol 54:549-64, Sep 85

16. Cathie, A.G.: The fascia of the body in relation to function and manip- ulative therapy. American Academy of Osteopathy Yearbook, Newark, Ohio, 1974 , pp. $81-4$

17. DeVries, H.A.: Electromyographic observations of the effects of static stretching upon muscular distress. Res Quart 32:468-79, 1961

18. Newham, D.J., et al.: Pain and fatigue after concentric and eccentric muscle contractions. Clin Sci 64:55-62, Jan 83

19. Bobbert, M.F., Hollander, A.P., and Huijing, P.A.: Factors in delayed onset muscular soreness of man. Med Sci Sports Exerc 18:75-81, Feb 86

Accepted for publication in November 1986. Updating, as necessary, has been done by the authors.

Mr. Molea, Mr. Murcek, Mr. Blanken, and Mr. Burns were student research associates at the Somatic Dysfunction Research Institute, Ohio University College of Osteopathic Medicine, Athens, Ohio. Dr. Chila is a professor and chairman, Department of Family Medicine, and Dr. Howell is an associate professor of biomedical science, OUCOM.

Dr. Howell, Ohio University College of Osteopathic Medicine, Irvine Hall, Athens, Ohio 45701-2979. 